\begin{tabular}{|c|c|}
\hline$A$ & $\begin{array}{c}\text { International Journal of Current Research } \\
\text { and Academic Review }\end{array}$ \\
\hline 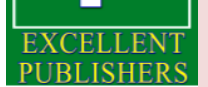 & 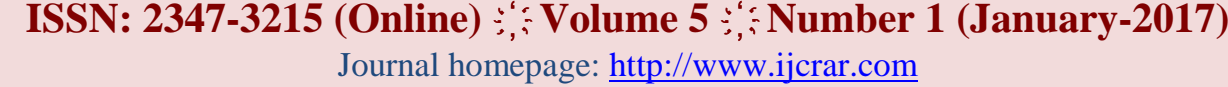 \\
\hline
\end{tabular}

doi: http://dx.doi.org/10.20546/ijcrar.2017.501.015

\title{
Geometrical classification and estimation of stresses of the joints in the ShewaSur- Chamchamal Anticline, NE Iraq
}

\author{
Duraid B. Deikran ${ }^{1}$ and Aryan S. Baban ${ }^{2} *$ \\ ${ }^{I}$ College of Agriculture, Kirkuk University, Iraq \\ ${ }^{2}$ Applied Geology Department, College of Science, Kirkuk University, Iraq \\ *Corresponding author.
}

\begin{abstract}
A classic joints survey have been executed on Shewa Sur-Chemchamal anticline seeking for geometrical classification of the joints and their causative stresses being the mentioned anticline not yet touched from structural point of view. Approximately, all type of joints in the mentioned anticline appeared:ab, bc, ac, hko1, hko2, hko3, hko4, okl, hol acute about a, hol acute about c and hol. Nearly, all estimated stress is of NE--SW trend which (Zagros type) and are comfortable with the nearby anticlines.
\end{abstract}

\section{Article Info}

Accepted: 15 January 2017

Available Online: 10 January 2017

\section{Keywords}

Shewa Sur-Anticline,

Geometrical Classification

\section{Introduction}

A classic method of joint study was used for execution this work. Field work was carried out in the form of isolated stations selected along lines perpendicular to Shewasur-Chem chemal anticline (study area) hinge area. In each station the joints were measured by compass and classified geometrically according to Hancook, 1985, then presented on stereonet.

It is worth to mention that joints appear only near the hinge line of the anticline.

The conjugate joints, among the measured joints, were used to estimate the paleo stress trend.

A lot of academic studies were carried out concerning joints in Iraq territory following the mentioned steps of work, Alazawi, 2010; Alkhatony, 2009; Al-Brifkani, 2008; AlSumaidaie, 2010; Al-Abdullah, 2009; Barno, 2011; Shihab, 2015. Al mentioned works illustrates that approximately all kinds of joints are existed and the estimated stress direction is nearly NE-SW.

In addition, there are many works, concerning present case study, outside of Iraq in general. Among these works are: Dyer, 1988; Lash \& Engelder, 2007; McConaughy \& Engelder, 2000; ZHAO and JACOBI, 1996.

The relation of joints and stress was discussed by ENOELDER \& Geiser (1980), they concluded from field studies on the Appalachian Plateau that regional systematic joints reflect the principal directions of the regional stress field which gave rise to the joint.

Moreover, joints are opening-mode fractures that propagate in the plane of s 1 and s 2 and normal to s 3 , and thus are sensitive indicators of the local stress eld orientation Dyer, 1988; Pollard and Aydin, 1988.

Systematic joints align parallel to the trend of the maximum horizontal stress (SH), they are used to 
construct regional paleo stress trajectories (Engelder and Geiser, 1980).

Furthermore, late-formed joints are often aligned parallel to the regional trend of the contemporary tectonic stress (modern-day $\mathrm{SH}$ ), and are thus used for mapping the orientation of neotectonic stress, Engelder,1982 and Hancock, 1991 and many others.

More than 800 measurements of joints were carried out to fulfill the target of this study. The main aim of the present study is to identify various types of joints and to extract the regional paleo stress in the study area.

\section{Location \& Geologic setting}

The study area is located about $70 \mathrm{~km}$. NE Kirkuk city, NE Iraq. The length of the studied anticline attains 75 $\mathrm{km}$., trending toward NW. Chem chamal city and Lesser Zab River located at southeastern and northwestern ends of the study area respectively, figure (1)

Tectonically, the study area lies within low folded zone of Zagros Fold-Thrust Belt, that consists of a series of widely spaced, low amplitude gentle folds trending NW SE but change gradually to $\mathrm{E}-\mathrm{W}$ as they extend northwestwards, Buday \& Jassim, 1987. This zone is characterized by the presence of regional detachment consists of Middle Miocene salt layers of Fat'ha Formation, that have caused decoupling of the surface structures from their subsurface counterparts (op cit), figure (2).

The study area is wide, gentle anticline and single plunging anticline in the southeastern end, figure (3). The lithology of rock units exposed in the study area is built up of alternating beds of gypsum, marl, claystone, sandstone and conglomerate represented by Miocene and Pliocene formations: Fat' ha (Middle Miocene, $370 \mathrm{~m}$. thick) in the core of anticline, Injana (Upper Miocene, 1266 thick), Mukdadiya (Pliocene, 840m. thick)) and Bia-Hassan (Pliocene, 906.5m. thick) which form the north western part of the study area figure (3).

Geometrical classification of joints (see table $1 \& 2$, figure (4) (5) and figure (3) whenever necessary)

As mentioned above, the present classification depends totally to Hancook, 1988. The following joint classes were distinguishes:

-ac class: The plane of this class parallel to tectonic axes $c$ and $a$ and perpendicular to $b$. In the NE limb this class appear in station $(54,43,24,20,5)$ with $84 \%$ while in the SW limb $(50,43,24,20,5)$ with $4.59 \%$.

-bc class: This class is parallel to the direction of dip. The line of intersection of this class is parallel to the bedding plane and strike of the beds. It appears in station $(14,5)$ with 2.3 in the NE limb while it appears in stations $(15,9)$ with $2.85 \%$.

-ab class: This class includes the bedding planes and all planes that are parallel to them. The joints appear only in station (16) in the SW limb with $1.26 \%$.

-hko class: This class is divided into two sub classes:

The first is hko acute about a: The planes of this subclass are acute about a axis. The acute angle ( 60) resulted from the intersection of plane of this subclass. The $b$ anda axes bisect the acute and obtuse angles respectively.

This class has two planes:

*hko1 acute about a that appear in the NE limb in stations $(28,22,16,6,1)$ with $4.61 \%$ while in the SW limb, they exist in the station $(50,42,41,26,17,11,10,9,4)$ with $11.72 \%$.

*hko2 acute about a existing in the NE limb in stations $((52,48,47,40,36,28,22,8)$ with 11.53 while in the SW limb the planes of hko2 acute about a appear in station $(34,19,17,15,7,3)$.

The second is the hko subclass acute about $\mathrm{b}\left(60^{\circ}\right)$, a and $b$ axes bisect the acute angle and obtuse angle of its planes respectively. This subclass comprises two planes:

* hko3 acute about b appears in stations in the NE $\operatorname{limb}(39,27,20,5,1)$ with $10.36 \%$. while in the SW limb they appear in stations $(49,38,34,30,23,10)$.

*hko4 acute about b appear in stations (40, 32, 31, 28, 5) in the NE limb with $8.84 \%$. While they appear in the SW limb in station $(30,23,21,11)$ with $6.33 \%$.

-okl class: The line of intersection of okl planes with bedding plane are parallel to a axis and perpendicular to fold axis. Only oklsubclass acute about c exist in the study area. The planes of this subclass make an angle 60 with bedding plane ang also make an acute angle with $\mathrm{c}$ axis ang obtuse angle withabout $b$ axis. 
In the NE limb this subclass appears instatio $(44,40,36$, $35,18,6)$ with $15.19 \%$ while in the SW limb it exists in the station $(53,51,49,45,4137,33,26,25,21,12,2)$ with $17.11 \%$.

holclass: The planes of this class are parallel to fold axis and intersect $\mathrm{a}$ and $\mathrm{c}$ axes. The line of intersections of this class are parallel to $b$ axis. It occurs in two subclasses:

*hol acute about a: The planes of this subclass make 60 about a axis and obtuse angle about $\mathrm{c}$ axis.Also make 30 with bedding plane. In the NE limb they appear in stations $(48,44,39,16,14)$ with $5.76 \%$ while in the SW limb they exist in stations $(15,7)$ with $2.21 \%$. hol acute about c: The plane of this class makes an acute angle about $\mathrm{c}$ axis and obtuse angle with a axis, moreover it makes 60 with bedding planes. It appears in the NE limb in stations $(53,51,45,38,3733,19,1310,9,7,4,3)$ with $11.92 \%$ while in the SW limbs it appears in stations $(53,51,45,38,37,33,19,13,10,9,7,4,3)$ with18.22\%.

hkl class: The planes of this class are always oblique to the tectonic axes. It appears in the NE limb in $(54,52,47,36,32,31,27,24,22,8,6)$ with $21.34 \%$ while in the SW limb it appears in stations $(46,42,41,38,37,29,25,21,17,13,4,3,2)$ with $20.91 \%$.

Table 1. Numerical details of the measured joints in NE limb

\begin{tabular}{|c|c|c|c|c|c|c|c|}
\hline $\begin{array}{l}\text { Classifi } \\
\text { cation }\end{array}$ & $\begin{array}{l}\text { Obtuse } \\
\text { and ob }\end{array}$ & $\begin{array}{l}\text { f joint plane } \\
\text { dding } \\
\text { ector trend. } \\
\text { e, } \\
\text { lunge }\end{array}$ & $\begin{array}{l}\text { Acute a } \\
\text { bedding : } \\
\text { angle, }\end{array}$ & $\begin{array}{l}\text { nt plane with } \\
\text { bisector trend. } \\
\text { trend/plunge }\end{array}$ & $\begin{array}{c}\text { Orientation } \\
\text { and plunge of } \\
\text { intersected line } \\
\text { with bedding } \\
\text { trend/plunge }\end{array}$ & $\begin{array}{c}\text { Average of } \\
\text { measurement of joint } \\
\text { cluster } \\
\text { Strike/dipdir/dip }\end{array}$ & \begin{tabular}{|} 
StationVlatitude \\
longitudel \\
Elevationlmean \\
bedding plane
\end{tabular} \\
\hline $\begin{array}{l}\mathrm{hko}_{3} \\
\mathrm{hko}_{1}\end{array}$ & $\begin{array}{l}96.4 \\
97.4\end{array}$ & $\begin{array}{l}109.9 / 43.6 \\
273.6 / 44.9\end{array}$ & $\begin{array}{l}83.6 \\
82.6\end{array}$ & $\begin{array}{l}293.8 / 46.4 \\
273.6 / 44.9\end{array}$ & $\begin{array}{c}111.9 / 02.0 \\
90 / 3.5\end{array}$ & $\begin{array}{c}292 / 202 / 88.222 \\
270.25 / 180.25 / 86.125\end{array}$ & $\begin{array}{c}1 / 35.4844 .32 / 470 \\
315 / 45 / 5\end{array}$ \\
\hline $\begin{array}{c}\text { hkl } \\
\text { okl }>\mathrm{c} \\
\mathrm{hko}_{1}\end{array}$ & $\begin{array}{l}100.2 \\
150.7 \\
154.1\end{array}$ & $\begin{array}{c}326.3 / 55 \\
6.5 / 78.4 \\
45.9 / 76.9\end{array}$ & $\begin{array}{l}79.8 \\
29.3 \\
25.9\end{array}$ & $\begin{array}{c}148.5 / 35 \\
205.9 / 12.3 \\
247.5 / 14.1\end{array}$ & $\begin{array}{c}327 / 1 \\
7.3,4 \\
47.1 / 5\end{array}$ & $\begin{array}{c}212.4 / 302.4 / 86.6 \\
288.28 / 198.28 / 87.14 \\
353.2 / 263.2 / 88.6 \\
266.5 / 176.5 / 86.75\end{array}$ & $\begin{array}{c}6 / 35.4944 .32 / 450 \\
315 / 47 / 5\end{array}$ \\
\hline $\begin{array}{c}\text { bc } \\
\text { hol>c } \\
\text { hol }>\text { a }\end{array}$ & $\begin{array}{l}100.7 \\
109.8 \\
161.5\end{array}$ & $\begin{array}{l}132.8 / 45.4 \\
301.2 / 59.9 \\
126.5 / 75.8\end{array}$ & $\begin{array}{l}79.3 \\
70.2 \\
18.5\end{array}$ & $\begin{array}{l}311.1 / 44.6 \\
121.1 / 30.1 \\
304.9 / 14.2\end{array}$ & $\begin{array}{c}312 / 9 \\
121.2 / 1 \\
306.4 / 4\end{array}$ & $\begin{array}{c}311.87 / 221.87 / 84.25 \\
121.16 / 31.66 / 65.16 \\
305.5 / 215.5 / 23.5\end{array}$ & $\begin{array}{c}14 / 35.47 \\
44.35 .5 / 620 \\
302 / 45 / 5\end{array}$ \\
\hline $\begin{array}{l}\text { hol }>c \\
\text { hol }>\text { a } \\
\text { hko }_{1}\end{array}$ & $\begin{array}{c}90 \\
163.7 \\
97\end{array}$ & $\begin{array}{c}312.2 / 49.9 \\
131 / 76.9 \\
58.7 / 46\end{array}$ & $\begin{array}{c}90 \\
16.3 \\
83\end{array}$ & $\begin{array}{l}134.2 / 40.1 \\
307.5 / 13.1 \\
247.3 / 44.2\end{array}$ & $\begin{array}{c}313 / 1 \\
310.8 / 8 \\
62.9 / 04.3\end{array}$ & $\begin{array}{c}133.13 / 43.13 / 85.06 \\
308.85 / 218.85 / 21.28 \\
243.2 / 333.2 / 85.6\end{array}$ & $\begin{array}{c}16 / 35.4644 .35 / 630 \\
302 / 47 / 5\end{array}$ \\
\hline $\begin{array}{l}\mathrm{okl}>\mathrm{c} \\
\mathrm{okl}>\mathrm{c}\end{array}$ & $\begin{array}{l}105.9 \\
102.7\end{array}$ & $\begin{array}{l}48.9 / 50.6 \\
29.6 / 51.3\end{array}$ & $\begin{array}{l}74.1 \\
77.3\end{array}$ & $\begin{array}{c}242 / 40.2 \\
223.8 / 39.6\end{array}$ & $\begin{array}{c}54.2 / 6.5 \\
35.2 / 7\end{array}$ & $\begin{array}{l}235.75 / 325.75 / 76.87 \\
216.75 / 126.75 / 77.83\end{array}$ & $\begin{array}{c}18 / 35.4544 .38 / 740 \\
302 / 47 / 7\end{array}$ \\
\hline $\begin{array}{c}\text { hkl } \\
\text { hkl } \\
\text { hko2 } \\
\text { hko1 }\end{array}$ & $\begin{array}{c}137.9 \\
138.1 \\
95 \\
98.8\end{array}$ & $\begin{array}{l}161.7 / 64.9 \\
348.3 / 72.7 \\
32.5 / 47.5 \\
89.3 / 45.3\end{array}$ & $\begin{array}{c}42.1 \\
41.9 \\
85 \\
81.2\end{array}$ & $\begin{array}{l}334.2 / 25.3 \\
180.5 / 17.7 \\
222.5 / 42.9 \\
274.7 / 44.9\end{array}$ & $\begin{array}{c}340.3 / 2.9 \\
349.4 / 3.5 \\
37.1 / 5 \\
92 / 2.7\end{array}$ & $\begin{array}{c}337.57 / 247.57 / 46.28 \\
173.85 / 83.85 / 38.42 \\
217.5 / 307.5 / 85.16 \\
272.2 / 182.2 / 85.4\end{array}$ & $\begin{array}{c}22 / 35.4344 .42 / 755 \\
305 / 47 / 5\end{array}$ \\
\hline $\begin{array}{c}\text { ac } \\
\text { hkl } \\
\text { hkl }\end{array}$ & $\begin{array}{c}95 \\
110.3 \\
100.8\end{array}$ & $\begin{array}{c}30 / 47.7 \\
117.5 / 50.2 \\
354.1 / 53.6\end{array}$ & $\begin{array}{c}85 \\
69.7 \\
79.2\end{array}$ & $\begin{array}{c}220 / 42.7 \\
298.8 / 39.8 \\
182.3 / 36.7\end{array}$ & $\begin{array}{c}34.6 / 5.0 \\
118.1 / 00.6 \\
357 / 3.9\end{array}$ & $\begin{array}{c}215 / 305 / 85 \\
298.22 / 208.22 / 74.66 \\
178 / 88 / 76.14\end{array}$ & $\begin{array}{c}24 / 35.4144 .46 / 645 \\
305 / 45 / 5\end{array}$ \\
\hline
\end{tabular}


Int.J.Curr.Res.Aca.Rev.2017; 5(1): 121-133

\begin{tabular}{|c|c|c|c|c|c|c|c|}
\hline $\begin{array}{c}\text { hko3 } \\
\text { hkl }\end{array}$ & $\begin{array}{c}104.7 \\
132\end{array}$ & $\begin{array}{l}87.9 / 45.8 \\
38.6 / 65.2\end{array}$ & $\begin{array}{c}75.3 \\
48\end{array}$ & $\begin{array}{l}276.6,44.5 \\
238.8 / 26.2\end{array}$ & $\begin{array}{l}92.2 / 4.4 \\
42.3 / 7.9\end{array}$ & $\begin{array}{c}272.78 / 182.78 / 82 \\
229.125 / 139.125 / 49\end{array}$ & $\begin{array}{c}27 / 35.4044 .48 / 600 \\
305 / 45 / 8\end{array}$ \\
\hline $\begin{array}{l}\mathrm{hko}_{4} \\
\mathrm{hko}_{2} \\
\mathrm{hko}_{1}\end{array}$ & $\begin{array}{l}104.6 \\
93.4 \\
97.1\end{array}$ & $\begin{array}{c}140.7 / 42.6 \\
6.3 / 50.9 \\
46.2 / 46.1\end{array}$ & $\begin{array}{l}75.4 \\
86.6 \\
82.5\end{array}$ & $\begin{array}{l}316.1 / 47.5 \\
205.1 / 40.7 \\
244 / 45.5\end{array}$ & $\begin{array}{c}318.2 / 2.3 \\
14 / 9.3 \\
55 / 9\end{array}$ & $\begin{array}{c}318 / 288 / 85.14 \\
195.16 / 285.16 / 83.16 \\
236 / 146 / 86\end{array}$ & $\begin{array}{c}28 / 35.4144 .50 / 605 \\
305 / 50 / 10\end{array}$ \\
\hline $\begin{array}{c}\mathrm{hko}_{4} \\
\mathrm{hkl}\end{array}$ & $\begin{array}{l}105.4 \\
119.1\end{array}$ & $\begin{array}{l}168.6 / 43.4 \\
339.9 / 69.2\end{array}$ & $\begin{array}{l}74.6 \\
60.9\end{array}$ & $\begin{array}{c}341.7 / 46.8 \\
168.2 / 21\end{array}$ & $\begin{array}{l}345 / 3.5 \\
341 / 2.8\end{array}$ & $\begin{array}{c}344.63 / 254.63 / 84 \\
163.2 / 73.2 / 51.3\end{array}$ & $\begin{array}{c}31 / 35.3844 .50 / 665 \\
325 / 40 / 10\end{array}$ \\
\hline $\begin{array}{c}\mathrm{hko}_{4} \\
\text { hkl }\end{array}$ & $\begin{array}{l}105.4 \\
142.6\end{array}$ & $\begin{array}{l}147.7 / 45.7 \\
115.3 / 65.2\end{array}$ & $\begin{array}{l}74.6 \\
37.4\end{array}$ & $\begin{array}{l}327.144 .3 \\
303.9 / 25.1\end{array}$ & $\begin{array}{c}327.4 / 00.3 \\
116.8 / 3.3\end{array}$ & $\begin{array}{l}327.35 / 237.35 / 81.64 \\
300.28 / 210.28 / 43.71\end{array}$ & $\begin{array}{c}32 / 35.3944 .51 / 640 \\
325 / 43 / 7\end{array}$ \\
\hline $\begin{array}{l}\mathrm{okl}>\mathrm{c} \\
\mathrm{okl}>\mathrm{c}\end{array}$ & $\begin{array}{l}100.2 \\
95.5\end{array}$ & $\begin{array}{l}60.3 / 49.1 \\
22.3 / 51.3\end{array}$ & $\begin{array}{l}79.8 \\
84.5\end{array}$ & $\begin{array}{c}254 / 41.7 \\
214.9 / 39.4\end{array}$ & $\begin{array}{l}66.2 / 6.9 \\
27.3 / 6.2\end{array}$ & $\begin{array}{l}247.31 / 337.31 / 81.25 \\
208.28 / 118.28 / 81.28\end{array}$ & $\begin{array}{c}35 / 35.3644 .52 / 705 \\
325 / 47 / 7\end{array}$ \\
\hline $\begin{array}{c}\mathrm{hkl} \\
\mathrm{okl}>\mathrm{c} \\
\mathrm{hkO}_{2}\end{array}$ & $\begin{array}{c}141.6 \\
101.9 \\
91.4\end{array}$ & $\begin{array}{c}66 / 67.2 \\
50 / 50.6 \\
21.9 / 52.6\end{array}$ & $\begin{array}{l}38.4 \\
78.1 \\
88.6\end{array}$ & $\begin{array}{l}281.7 / 27.4 \\
259.1 / 43.2 \\
229.5 / 40.8\end{array}$ & $\begin{array}{l}72.2 / 14.4 \\
62.6 / 14.9 \\
32.8 / 13.9\end{array}$ & $\begin{array}{c}267.14 / 177.14 / 44.71 \\
245.2 / 335.2 / 80.4 \\
214.5 / 304.5 / 83.2\end{array}$ & $\begin{array}{c}36 / 35.3744 .53 / 710 \\
325 / 45 / 15\end{array}$ \\
\hline $\begin{array}{l}\mathrm{hko}_{3} \\
\text { hol>a } \\
\text { hol>c }\end{array}$ & $\begin{array}{c}117.1 \\
165.5 \\
95.9\end{array}$ & $\begin{array}{l}116.2 / 49.6 \\
163.7 / 87.8 \\
134.3 / 38.1\end{array}$ & $\begin{array}{l}62.9 \\
14.5 \\
84.1\end{array}$ & $\begin{array}{c}304.9 / 40.7 \\
288 / 3.9 \\
317.5 / 52.0\end{array}$ & $\begin{array}{l}119.9 / 4.3 \\
343.6 / 3.2 \\
136.2 / 1.5\end{array}$ & $\begin{array}{c}301.28 / 211.28 / 72 \\
196 / 106 / 6 \\
136.14 / 46.14 / 86\end{array}$ & $\begin{array}{c}39 / 35.3244 .54 / 666 \\
325 / 47 / 10\end{array}$ \\
\hline $\begin{array}{l}\mathrm{hko}_{2} \\
\mathrm{hko}_{4} \\
\mathrm{okl}>\mathrm{c}\end{array}$ & $\begin{array}{c}92.7 \\
100.4 \\
98\end{array}$ & $\begin{array}{c}204.8 / 42.5 \\
167.9 / 43.7 \\
58.8 / 48.2\end{array}$ & $\begin{array}{c}87.3 \\
79.6 \\
82\end{array}$ & $\begin{array}{c}13.5 / 48 \\
343 / 46.4 \\
252.6 / 42.7\end{array}$ & $\begin{array}{c}18.6 / 5.6 \\
345.3 / 2.4 \\
65 / 6.9\end{array}$ & $\begin{array}{l}198.71 / 288.71 / 88.57 \\
345.16 / 255.16 / 86.16 \\
245.85 / 335.85 / 83.28\end{array}$ & $\begin{array}{c}40 / 35.3444 .56 / 740 \\
325 / 50 / 7\end{array}$ \\
\hline $\begin{array}{c}\text { ac } \\
\text { hol }>\mathrm{c}\end{array}$ & $\begin{array}{l}95.7 \\
96.6\end{array}$ & $\begin{array}{l}35.8 / 49.8 \\
132.8 / 36.4\end{array}$ & $\begin{array}{l}84.3 \\
83.4\end{array}$ & $\begin{array}{l}239.4 / 42.7 \\
315.3 / 53.6\end{array}$ & $\begin{array}{l}46.1 / 12.0 \\
134.4 / 1.2\end{array}$ & $\begin{array}{c}227.5 / 137.5 / 83.6 \\
134.3 / 44.3 / 84.7\end{array}$ & $\begin{array}{c}43 / 35.3144 .56 / 680 \\
320 / 38 / 12\end{array}$ \\
\hline $\begin{array}{l}\text { hol>a } \\
\text { okl>c } \\
\text { okl }>c\end{array}$ & $\begin{array}{c}162 \\
104.1 \\
98\end{array}$ & $\begin{array}{c}151.9 / 84.3 \\
41.2 / 52.8 \\
18.1 / 55.8\end{array}$ & $\begin{array}{c}18 \\
75.9 \\
82\end{array}$ & $\begin{array}{c}303.3 / 6.5 \\
250.7 / 41.1 \\
226.6 / 37.7\end{array}$ & $\begin{array}{c}331.5 / 3.1 \\
52.9 / 15 \\
27.9 / 13.9\end{array}$ & $\begin{array}{c}194.5 / 104.5 / 4.5 \\
236.37 / 326.37 / 77.12 \\
211.2 / 121.2 / 76.8\end{array}$ & $\begin{array}{c}44 / 35.3244 .57 / 773 \\
320 / 47 / 15\end{array}$ \\
\hline $\begin{array}{c}\mathrm{hkl} \\
\mathrm{hkO}_{2}\end{array}$ & $\begin{array}{c}139.7 \\
91.9\end{array}$ & $\begin{array}{l}156.4 / 65.1 \\
11.8 / 48.9\end{array}$ & $\begin{array}{l}40.3 \\
88.1\end{array}$ & $\begin{array}{l}332.8 / 25 \\
200 / 41.4\end{array}$ & $\begin{array}{c}335.8 / 1.4 \\
15.4 / 4.1\end{array}$ & $\begin{array}{l}334.4 / 244.4 / 45.1 \\
195.7 / 285.7 / 85.3\end{array}$ & $\begin{array}{c}47 / 35.2944 .57 / 650 \\
320 / 45 / 5\end{array}$ \\
\hline $\begin{array}{l}\text { hol>c } \\
\text { hko } \\
\text { hol>a }\end{array}$ & $\begin{array}{c}133.3 \\
90.4 \\
168.5\end{array}$ & $\begin{array}{c}121.7 / 59 \\
199.9 / 40.8 \\
159.6 / 88.2\end{array}$ & $\begin{array}{l}46.7 \\
89.6 \\
11.5\end{array}$ & $\begin{array}{c}307 / 31.1 \\
7.1 / 49.9 \\
283 / 3.2\end{array}$ & $\begin{array}{c}123.1 / 02.3 \\
12.5 / 6.4 \\
339.5 / 02.7\end{array}$ & $\begin{array}{c}304.81 / 214.81 / 54.36 \\
193 / 283 / 85.6 \\
193.6 / 103.6 / 4.8\end{array}$ & $\begin{array}{c}48 / 35.3044 .59 / 680 \\
320 / 45 / 8\end{array}$ \\
\hline $\begin{array}{r}\mathrm{hkl} \\
\mathrm{hkl} \\
\mathrm{hko}_{2}\end{array}$ & $\begin{array}{l}131.3 \\
150.9 \\
97.5\end{array}$ & $\begin{array}{c}112 / 61 \\
6 / 78.5 \\
11.1 / 51.4\end{array}$ & $\begin{array}{l}48.7 \\
29.1 \\
82.5\end{array}$ & $\begin{array}{c}296.4 / 29 \\
205.5 / 12.1 \\
199.9 / 38.9\end{array}$ & $\begin{array}{c}113 / 1.9 \\
6.8 / 3.9 \\
14.5 / 4.3\end{array}$ & $\begin{array}{c}294.42 / 204.42 / 53.42 \\
194.83 / 104.83 / 26.33 \\
195.28 / 285.28 / 80\end{array}$ & $\begin{array}{c}52 / 35.5344 .26 / 428 \\
315 / 48 / 5\end{array}$ \\
\hline $\begin{array}{c}\text { hkl } \\
\text { ac }\end{array}$ & $\begin{array}{c}123.8 \\
93.9\end{array}$ & $\begin{array}{c}25.2 / 65 \\
44.9 / 47.1\end{array}$ & $\begin{array}{l}56.2 \\
86.1\end{array}$ & $\begin{array}{c}224.1 / 26.2 \\
240.8 / 44\end{array}$ & $\begin{array}{c}28.7 / 7.5 \\
52.4 / 8\end{array}$ & $\begin{array}{l}214.2 / 304.2 / 53.7 \\
232.9 / 142.9 / 86.5\end{array}$ & $\begin{array}{c}54 / 34.5444 .24 / 470 \\
320 / 45 / 8\end{array}$ \\
\hline
\end{tabular}


Table 2. Numerical details of the measured joints in SW limb

\begin{tabular}{|c|c|c|c|c|c|c|c|}
\hline \multirow{2}{*}{$\begin{array}{c}\begin{array}{c}\text { Classific } \\
\text { ation }\end{array} \\
\\
\\
\text { hkl } \\
\text { okl>c } \\
\text { hkl }\end{array}$} & \multicolumn{2}{|c|}{$\begin{array}{l}\text { Obtuse angle of joint plane } \\
\text { with bedding } \\
\text { and obtuse bisector trend. } \\
\text { angle, trend/plunge }\end{array}$} & \multicolumn{2}{|c|}{$\begin{array}{l}\text { Acute angle of joint plane with } \\
\text { bedding and acute bisector } \\
\text { trend. } \\
\text { angle, trend/plunge }\end{array}$} & \multirow{2}{*}{ 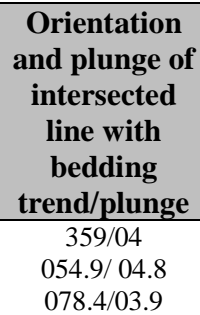 } & \multirow{2}{*}{ 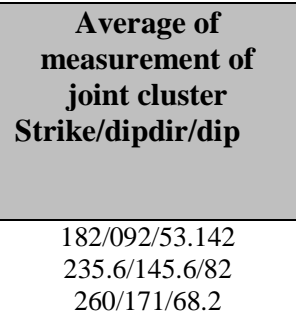 } & \multirow{2}{*}{$\begin{array}{c}\begin{array}{c}\text { Stationlatitude } \\
\text { longitude } \\
\text { Elevationlmean } \\
\text { bedding plane }\end{array} \\
2 / 35.4744 .32 / 460 \\
310 / 220 / 05\end{array}$} \\
\hline & $\begin{array}{c}124 \\
157.29 \\
114.52\end{array}$ & $\begin{array}{l}357.4 / 65.2 \\
050.6 / 48.6 \\
075.6 / 54.5\end{array}$ & $\begin{array}{c}56 \\
22.71 \\
65.48\end{array}$ & $\begin{array}{r}187.3 / 25.1 \\
240.3 / 41.9 \\
263.9 / 35.8\end{array}$ & & & \\
\hline $\begin{array}{c}\mathrm{hkO}_{2} \\
\mathrm{hkl} \\
\mathrm{hol}>\mathrm{c}\end{array}$ & $\begin{array}{c}90.62 \\
135.32 \\
96.48\end{array}$ & $\begin{array}{l}055.5 / 45.3 \\
195.6 / 64.0 \\
191.9 / 43.3\end{array}$ & $\begin{array}{c}89.38 \\
42.6 \\
82.7\end{array}$ & $\begin{array}{l}252.4 / 46.0 \\
356.9 / 27.2 \\
358.2 / 47.5\end{array}$ & $\begin{array}{l}064.1 / 08.5 \\
011.9 / 07.6 \\
004.6 / 06.9\end{array}$ & $\begin{array}{c}255.4 / 345.4 / 87.8 \\
365 / 275 / 48 \\
364.4 / 274.4 / 88.5\end{array}$ & $\begin{array}{c}3 / 35.46 .44 .31 .36 / 594 \\
315 / 225 / 09\end{array}$ \\
\hline $\begin{array}{c}\mathrm{hko}_{1} \\
\text { hol>c } \\
\text { hkl }\end{array}$ & $\begin{array}{l}106.43 \\
131.43 \\
101.98\end{array}$ & $\begin{array}{l}350.8 / 53.6 \\
284.5 / 68.4 \\
084.0 / 47.4\end{array}$ & $\begin{array}{c}73.57 \\
48.8 \\
80.7\end{array}$ & $\begin{array}{r}175.4 / 36.5 \\
101.3 / 21.6 \\
267.8 / 42.7\end{array}$ & $\begin{array}{l}352.4 / 02.2 \\
104.0 / 01.1 \\
085.8 / 01.9\end{array}$ & $\begin{array}{l}173 / 83.3 / 75 \\
302 / 052 / 46 \\
266 / 176 / 83\end{array}$ & $\begin{array}{c}/ 35.4644 .32 / 585 \\
305 / 215 / 09\end{array}$ \\
\hline $\begin{array}{c}\mathrm{hol}>\mathrm{c} \\
\mathrm{hko}_{2} \\
\mathrm{hol}>\mathrm{a}\end{array}$ & $\begin{array}{c}104.5 \\
91.8 \\
134.3\end{array}$ & $\begin{array}{l}155.1 / 44.3 \\
025.3 / 50.1 \\
130.9 / 59.6\end{array}$ & $\begin{array}{l}75.5 \\
88.2 \\
45.7\end{array}$ & $\begin{array}{l}333.9 / 45.7 \\
219.4 / 40.8 \\
316.5 / 30.5\end{array}$ & $\begin{array}{l}334.5 / 00.6 \\
031.2 / 07.0 \\
132.3 / 02.4\end{array}$ & $\begin{array}{c}334.428 / 254.428 / 83.428 \\
211.857 / 121.857 / 84.42 \\
314.166 / 224.166 / 53.333\end{array}$ & $\begin{array}{c}7 / 35.48 \quad 44.30 / 503 \\
330 / 225 / 08\end{array}$ \\
\hline $\begin{array}{l}\mathrm{hko}_{1} \\
\mathrm{bc} \\
\mathrm{hol}>\mathrm{c}\end{array}$ & $\begin{array}{c}92.27 \\
94 \\
98\end{array}$ & $\begin{array}{l}008.6 / 49.0 \\
135.4 / 41.5 \\
134.3 / 45.0\end{array}$ & $\begin{array}{c}87.73 \\
86 \\
82.0\end{array}$ & $\begin{array}{r}195.7 / 41.2 \\
314.7 / 48.5 \\
313.7 / 45.0 \\
\end{array}$ & $\begin{array}{l}011.6 / 03.5 \\
315.0 / 00.3 \\
314.0 / 00.3\end{array}$ & $\begin{array}{l}192 / 102 / 84 \\
128 / 045 / 87 \\
314 / 224 / 86\end{array}$ & $\begin{array}{c}9 / 35.50 \quad 44.28 / 339 \\
310 / 220 / 04\end{array}$ \\
\hline $\begin{array}{r}\mathrm{hko}_{1} \\
\mathrm{hko}_{3} \\
\mathrm{hol}>\mathrm{c}\end{array}$ & $\begin{array}{c}98.02 \\
92.09 \\
104.04\end{array}$ & $\begin{array}{l}015.8 / 52.8 \\
181.1 / 40.1 \\
135.0 / 45.5\end{array}$ & $\begin{array}{l}81.98 \\
87.91 \\
75.96\end{array}$ & $\begin{array}{r}208.9 / 38.0 \\
351.8 / 50.3 \\
315.0 / 44.5\end{array}$ & $\begin{array}{l}020.6 / 06.4 \\
355.6 / 04.6 \\
315.0 / 00.0\end{array}$ & $\begin{array}{c}202 / 112 / 78 \\
176 / 86 / 85 \\
315 / 255 / 82\end{array}$ & $\begin{array}{c}10 / 35.4944 .27 / 450 \\
315 / 225 / 12\end{array}$ \\
\hline $\begin{array}{l}\mathrm{hko}_{4} \\
\mathrm{hko}_{1}\end{array}$ & $\begin{array}{l}94.72 \\
93.42\end{array}$ & $\begin{array}{l}123.1 / 38.2 \\
202.9 / 40.7\end{array}$ & $\begin{array}{l}85.28 \\
86.58\end{array}$ & $\begin{array}{l}308.1 / 51.9 \\
006.7 / 50.5\end{array}$ & $\begin{array}{l}126.2 / 02.4 \\
013.4 / 08.1\end{array}$ & $\begin{array}{l}126 / 036 / 86 \\
194 / 104 / 86\end{array}$ & $\begin{array}{c}11 / 35.5044 .27 / 405 \\
320 / 230 / 1\end{array}$ \\
\hline $\begin{array}{l}\mathrm{okl}>\mathrm{c} \\
\mathrm{okl}>\mathrm{c}\end{array}$ & $\begin{array}{l}108.99 \\
107.46\end{array}$ & $\begin{array}{l}009.7 / 59.9 \\
046.7 / 49.6\end{array}$ & $\begin{array}{l}71.01 \\
72.54\end{array}$ & $\begin{array}{l}239.8 / 42.2 \\
273.9 / 51.4 \\
\end{array}$ & $\begin{array}{l}026.4 / 26.5 \\
071.1 / 25.9\end{array}$ & $\begin{array}{l}212 / 302 / 79 \\
255 / 165 / 82 \\
\end{array}$ & $\begin{array}{c}12 / 35.4944 .25 / 340 \\
317 / 227 / 28\end{array}$ \\
\hline $\begin{array}{c}\text { hkl } \\
\text { hol>c } \\
\text { hol>c }\end{array}$ & $\begin{array}{l}149.5 \\
96.18 \\
95.71\end{array}$ & $\begin{array}{l}189.6 / 71.0 \\
030.3 / 48.4 \\
052.0 / 47.9\end{array}$ & $\begin{array}{c}30.5 \\
83.82 \\
84.29\end{array}$ & $\begin{array}{l}219.9 / 42.1 \\
219.9 / 42.1 \\
241.9 / 42.5\end{array}$ & $\begin{array}{l}008.4 / 03.7 \\
034.6 / 04.8 \\
056.5 / 05.0\end{array}$ & $\begin{array}{l}363 / 273 / 35 \\
215 / 305 / 85 \\
237 / 147 / 84\end{array}$ & $\begin{array}{c}13 / 35.4644 .33 / 566 \\
320 / 230 / 05\end{array}$ \\
\hline $\begin{array}{c}\mathrm{bc} \\
\mathrm{hol}>\mathrm{a} \\
\mathrm{hkO}_{2}\end{array}$ & $\begin{array}{c}92.85 \\
148.95 \\
90.84\end{array}$ & $\begin{array}{l}137.0 / 30.6 \\
102.7 / 60.6 \\
064.1 / 47.9\end{array}$ & $\begin{array}{l}87.15 \\
31.05 \\
89.16\end{array}$ & $\begin{array}{r}313.9 / 59.5 \\
297.7 / 30.3 \\
269.5 / 45.1\end{array}$ & $\begin{array}{c}314.7 / 01.3 \\
106.4 / 06.5 \\
076.2,13.0\end{array}$ & $\begin{array}{l}135 / 045 / 77 \\
315 / 225 / 45 \\
259 / 349 / 78\end{array}$ & $\begin{array}{c}15 / 35.4544 .35 / 532 \\
310 / 220 / 16\end{array}$ \\
\hline $\begin{array}{l}\mathrm{hko}_{1} \\
\mathrm{hko}_{2}\end{array}$ & $\begin{array}{c}102.31 \\
94.62\end{array}$ & $\begin{array}{l}006.7 / 53.4 \\
061.3 / 48.6\end{array}$ & $\begin{array}{l}77.69 \\
85.38\end{array}$ & $\begin{array}{l}195.7 / 36.9 \\
250.4 / 41.8\end{array}$ & $\begin{array}{l}009.9 / 04.3 \\
065.3 / 04.5\end{array}$ & $\begin{array}{l}191 / 101 / 76 \\
246 / 336 / 81\end{array}$ & $\begin{array}{c}17 / 35.4544 .36 / 600 \\
310 / 220 / 10\end{array}$ \\
\hline $\begin{array}{c}\mathrm{hkl} \\
\mathrm{hko}_{4} \\
\mathrm{okl}>\mathrm{c}\end{array}$ & $\begin{array}{c}130.21 \\
99 \\
108.56\end{array}$ & $\begin{array}{c}066.8 / 66.4 \\
308.8 / 57.0 \\
047.8\end{array}$ & $\begin{array}{c}49.3 \\
81 \\
71.44\end{array}$ & $\begin{array}{l}263.7 / 24.6 \\
127.0 / 33.0 \\
241.9 / 38.5\end{array}$ & $\begin{array}{l}069.6 / 06.4 \\
128.3 / 00.8 \\
053.1 / 06.9\end{array}$ & $\begin{array}{l}256 / 346 / 45 \\
128 / 045 / 73 \\
235 / 145 / 75 \\
\end{array}$ & $\begin{array}{c}21 / 35.4244 .41 / 790 \\
315 / 225 / 09\end{array}$ \\
\hline $\begin{array}{c}\mathrm{hko}_{3} \\
\mathrm{hko}_{4} \\
\mathrm{ac}\end{array}$ & $\begin{array}{l}93.96 \\
92.64 \\
93.84\end{array}$ & $\begin{array}{l}319.9 / 00.6 \\
104.4 / 39.5 \\
028.3 / 47.0\end{array}$ & $\begin{array}{l}86.04 \\
87.36 \\
86.16\end{array}$ & $\begin{array}{l}319.9 / 00.6 \\
290.9 / 50.7 \\
222.0 / 43.8\end{array}$ & $\begin{array}{l}319.9 / 00.6 \\
319.9 / 00.6 \\
319.9 / 00.6\end{array}$ & $\begin{array}{l}140 / 050 / 85 \\
108 / 018 / 85 \\
215 / 305 / 88\end{array}$ & $\begin{array}{c}23 / 35.40 \quad 44.45 / 710 \\
315 / 225 / 07\end{array}$ \\
\hline $\begin{array}{c}\mathrm{hkl} \\
\mathrm{okl}>\mathrm{c}\end{array}$ & $\begin{array}{l}154.22 \\
106.81\end{array}$ & $\begin{array}{l}163.7 / 68.5 \\
055.2 / 48.7\end{array}$ & $\begin{array}{l}25.78 \\
73.19\end{array}$ & $\begin{array}{l}328.5 / 22.2 \\
253.5 / 42.7\end{array}$ & $\begin{array}{l}341.6 / 05.3 \\
063.4 / 09.2\end{array}$ & $\begin{array}{l}334 / 244 / 35 \\
245 / 155 / 80\end{array}$ & $\begin{array}{c}25 / 35.3744 .45 / 745 \\
310 / 220 / 10\end{array}$ \\
\hline $\begin{array}{c}\mathrm{ab} \\
\mathrm{okl}>\mathrm{c} \\
\mathrm{hko}_{1}\end{array}$ & $\begin{array}{l}173.47 \\
98.25 \\
92.35\end{array}$ & $\begin{array}{l}154.8 / 82.7 \\
011.8 / 48.9 \\
203.7 / 43.4 \\
\end{array}$ & $\begin{array}{c}6.53 \\
81.75 \\
87.65 \\
\end{array}$ & $\begin{array}{l}321.7 / 07.5 \\
200.6 / 41.5 \\
014.7 / 47.0 \\
\end{array}$ & $\begin{array}{l}334.6 / 01.7 \\
015.6 / 04.4 \\
018.9 / 04.5 \\
\end{array}$ & $\begin{array}{c}325 / 235 / 10 \\
196 / 286 / 85 \\
199 / 109 / 88.6 \\
\end{array}$ & $\begin{array}{c}26 / 35.3944 .47 / 705 \\
315 / 225 / 05\end{array}$ \\
\hline hkl & 108.95 & $342.4 / 60.4$ & 71.05 & $168.0 / 29.7$ & $343.8 / 02.4$ & $165 / 75.4 / 63.7$ & $\begin{array}{c}29 / 35.3644 .46 / 730 \\
315 / 225 / 05 \\
\end{array}$ \\
\hline $\begin{array}{l}\mathrm{hko}_{4} \\
\mathrm{hko}_{3}\end{array}$ & $\begin{array}{c}90.62 \\
105.84\end{array}$ & $\begin{array}{c}280.5 / 51.6 \\
150.1 / 45 \\
\end{array}$ & $\begin{array}{l}89.38 \\
74.16 \\
\end{array}$ & $\begin{array}{l}090.6 / 38.8 \\
327.9 / 44.2 \\
\end{array}$ & $\begin{array}{l}096.7 / 04.8 \\
329.0 / 01.1 \\
\end{array}$ & $\begin{array}{c}289 / 010 / 82.2 \\
328.85 / 238.85 / 81.42 \\
\end{array}$ & $\begin{array}{c}30 / 35.3744 .48 / 740 \\
320 / 230 / 07 \\
\end{array}$ \\
\hline $\begin{array}{l}\text { okl }>\mathrm{c} \\
\text { hol }>\mathrm{c}\end{array}$ & $\begin{array}{l}109.54 \\
116.18\end{array}$ & $\begin{array}{l}253.4 / 38.2 \\
315.9 / 69.2\end{array}$ & $\begin{array}{l}70.46 \\
63.82\end{array}$ & $\begin{array}{r}253.4 / 38.2 \\
133.6 / 20.8\end{array}$ & $\begin{array}{l}060.7 / 09.8 \\
135.6 / 00.8\end{array}$ & $\begin{array}{c}243.8 / 153.8 / 72.7 \\
135.8 / 045 / 51.5\end{array}$ & $\begin{array}{c}33 / 35.34 \quad 44.48 / 760 \\
320 / 230 / 10\end{array}$ \\
\hline $\begin{array}{l}\mathrm{hko}_{2} \\
\mathrm{hko}_{3}\end{array}$ & $\begin{array}{l}93.52 \\
96.12\end{array}$ & $\begin{array}{l}069.4 / 340.8 \\
340.8 / 57.6\end{array}$ & $\begin{array}{l}86.48 \\
83.88\end{array}$ & $\begin{array}{l}266.0 / 46.5 \\
171.9 / 32.9\end{array}$ & $\begin{array}{l}078.0 / 08.4 \\
344.0 / 05.0\end{array}$ & $\begin{array}{l}258.5 / 348.5 / 86.35 \\
165.5 / 075.5 / 73.75\end{array}$ & $\begin{array}{c}34 / 35.3544 .50 / 785 \\
315 / 225 / 10\end{array}$ \\
\hline $\begin{array}{c}\text { hol }>\mathrm{c} \\
\mathrm{okl}>\mathrm{c} \\
\mathrm{hkl}\end{array}$ & $\begin{array}{l}153.23 \\
108.56 \\
142.98\end{array}$ & $\begin{array}{l}333.0 / 82.3 \\
050.5 / 53.0 \\
078.6 / 68.7\end{array}$ & $\begin{array}{l}26.77 \\
71.44 \\
37.02\end{array}$ & $\begin{array}{r}167.6 / 07.9 \\
240.5 / 37.4 \\
269.8 / 21.7\end{array}$ & $\begin{array}{l}333.2 / 02.0 \\
054.1 / 04.8 \\
080.1 / 03.8\end{array}$ & $\begin{array}{c}158.66 / 68.66 / 20 \\
235.6 / 145.6 / 73.2 \\
264.7 / 174.7 / 39.7\end{array}$ & $\begin{array}{c}37 / 35.3144 .50 / 720 \\
310 / 220 / 05\end{array}$ \\
\hline $\begin{array}{c}\mathrm{hkl} \\
\mathrm{hol}>\mathrm{c} \\
\mathrm{hko}_{3}\end{array}$ & $\begin{array}{c}167.21 \\
115.69 \\
102.3 \\
\end{array}$ & $\begin{array}{l}176.2 / 79.3 \\
009.0 / 57.9 \\
341.5,55.6\end{array}$ & $\begin{array}{c}13.19 \\
63.31 \\
77.7 \\
\end{array}$ & $\begin{array}{l}338.8 / 11.2 \\
198.2 / 32.4 \\
166.5,34.5\end{array}$ & $\begin{array}{l}355.5 / 03.3 \\
011.6 / 04.2 \\
343.1 / 02.4 \\
\end{array}$ & $\begin{array}{c}345.4 / 250.4 / 17.9 \\
193.4 / 283.6 / 67.2 \\
163.8 / 73.28 / 73.28 \\
\end{array}$ & $\begin{array}{c}38 / 35.3244 .52 / 680 \\
315 / 215 / 05\end{array}$ \\
\hline $\begin{array}{c}\mathrm{hkl} \\
\mathrm{okl}>\mathrm{c} \\
\mathrm{hko}_{1}\end{array}$ & $\begin{array}{c}135 \\
104.7 \\
99.4\end{array}$ & $\begin{array}{c}345.7 / 74.7 \\
035.0 / 54.0 \\
2.0 / 55.5\end{array}$ & $\begin{array}{c}45 \\
75.3 \\
80.6\end{array}$ & $\begin{aligned} 179.7 / 15.8 \\
231.3 / 37.1 \\
194.2 / 35.2\end{aligned}$ & $\begin{array}{c}346.7 / 03.6 \\
40.8 / 07.9 \\
6.0 / 05.8\end{array}$ & $\begin{array}{c}171.375 / 081.375 / 38 \\
223 / 313 / 74.2 \\
187.5 / 097.5 / 75.125\end{array}$ & $\begin{array}{c}41 / 35.2944 .52 / 670 \\
320 / 227 / 10\end{array}$ \\
\hline
\end{tabular}




\begin{tabular}{|c|c|c|c|c|c|c|c|}
\hline $\begin{array}{c}\text { hkl } \\
\text { ac } \\
\text { hko }_{1}\end{array}$ & $\begin{array}{c}139.96 \\
98.18 \\
102.81\end{array}$ & $\begin{array}{c}345.4 / 75.0 \\
039.8 / 49.1 \\
002.4 / 56.0\end{array}$ & $\begin{array}{l}40.04 \\
81.82 \\
77.19\end{array}$ & $\begin{array}{l}181.8 / 15.6 \\
235.8 / 42.0 \\
195.9 / 34.8\end{array}$ & $\begin{array}{l}346.6 / 04.2 \\
046.8 / 08.0 \\
006.7 / 06.3\end{array}$ & $\begin{array}{c}172.14 / 082.14 / 37.14 \\
227.42 / 137.42 / 86.03 \\
188.57 / 098.57 / 73.57\end{array}$ & $\begin{array}{c}42 / 35.3044 .54 / 610 \\
315 / 225 / 10\end{array}$ \\
\hline $\begin{array}{l}\text { hol }>\mathrm{c} \\
\text { okl }>\mathrm{c}\end{array}$ & $\begin{array}{l}145.72 \\
118.87\end{array}$ & $\begin{array}{l}336.2 / 80.3 \\
045.7 / 59.5\end{array}$ & $\begin{array}{l}34.28 \\
61.13\end{array}$ & $\begin{array}{l}173.6 / 10.2 \\
243.5 / 31.8\end{array}$ & $\begin{array}{l}336.7 / 03.0 \\
050.5 / 08.0\end{array}$ & $\begin{array}{c}152.58 / 073.58 / 27 \\
234.87 / 144.87 / 61.25\end{array}$ & $\begin{array}{c}45 / 35.2744 .53 / 645 \\
315 / 225 / 08\end{array}$ \\
\hline $\begin{array}{l}\text { hkl } \\
\text { hkl } \\
\text { hkl }\end{array}$ & $\begin{array}{l}151.27 \\
147.08 \\
125.63\end{array}$ & $\begin{array}{l}079.9 / 73.7 \\
057.1 / 75.3 \\
331.2 / 61.0\end{array}$ & $\begin{array}{l}28.73 \\
32.92 \\
54.37 \\
\end{array}$ & $\begin{array}{r}273.6 / 16.8 \\
255.8 / 15.5 \\
155.6 / 29.0\end{array}$ & $\begin{array}{l}081.0 / 03.8 \\
058.4 / 04.7 \\
332.2 / 01.9\end{array}$ & $\begin{array}{c}267.66 / 177.66 / 29.7 \\
247.25 / 337.25 / 28.25 \\
153.2 / 243.2 / 62.6\end{array}$ & $\begin{array}{c}46 / 35.2844 .56 / 625 \\
310 / 220 / 05\end{array}$ \\
\hline $\begin{array}{l}\mathrm{hko}_{3} \\
\mathrm{okl}>\mathrm{c}\end{array}$ & $\begin{array}{c}96.8 \\
104.9\end{array}$ & $\begin{array}{c}312.3 / 55.3 \\
48.6 / 50.1\end{array}$ & $\begin{array}{l}83.2 \\
75.1\end{array}$ & $\begin{array}{l}135.2 / 34.8 \\
241.7 / 40.6\end{array}$ & $\begin{array}{c}313.2 / 01.4 \\
54.1 / 06.5\end{array}$ & $\begin{array}{c}133.58 / 043.58 / 76.33 \\
235.5 / 145.5 / 77.83\end{array}$ & $\begin{array}{c}49 / 35.4344 .34 / 700 \\
302 / 225 / 07\end{array}$ \\
\hline $\begin{array}{c}\mathrm{hko}_{1} \\
\text { ac }\end{array}$ & $\begin{array}{l}98.3 \\
93.6\end{array}$ & $\begin{array}{l}059.6 / 47.7 \\
038.8 / 47.1\end{array}$ & $\begin{array}{l}81.7 \\
86.4\end{array}$ & $\begin{array}{l}249.0 / 42.7 \\
228.8 / 43.3\end{array}$ & $\begin{array}{l}063.9 / 04.7 \\
043.5 / 05.0\end{array}$ & $\begin{array}{c}244.444 / 334.444 / 83.3 \\
223.8 / 313.8 / 86.3\end{array}$ & $\begin{array}{c}50 / 35.4144 .38 / 770 \\
305 / 225 / 6\end{array}$ \\
\hline $\begin{array}{l}\text { hol }>\mathrm{c} \\
\mathrm{okl}>\mathrm{c}\end{array}$ & $\begin{array}{l}106.04 \\
114.55\end{array}$ & $\begin{array}{l}091.1 / 47.0 \\
196.3 / 49.6\end{array}$ & $\begin{array}{l}73.96 \\
65.45\end{array}$ & $\begin{array}{l}284.1 / 43.8 \\
000.6 / 41.5\end{array}$ & $\begin{array}{l}097.3 / 06.6 \\
009.6 / 07.9\end{array}$ & $\begin{array}{c}278.5 / 188.5 / 79.3 \\
367.54 / 227.54 / 75.72\end{array}$ & $\begin{array}{c}51 / 35.5344 .25 / 415 \\
318 / 228 / 10\end{array}$ \\
\hline $\begin{array}{l}\text { okl }>c \\
\text { hol }>\text { c }\end{array}$ & $\begin{array}{c}99.22 \\
124.01\end{array}$ & $\begin{array}{l}038.1 / 50.3 \\
316.2 / 75.4\end{array}$ & $\begin{array}{l}80.78 \\
55.99\end{array}$ & $\begin{array}{l}247.2 / 43.6 \\
137.5 / 14.6\end{array}$ & $\begin{array}{l}050.9 / 14.9 \\
316.3 / 00.3\end{array}$ & $\begin{array}{l}233.2 / 133.2 / 81.6 \\
136.6 / 046.6 / 44.2\end{array}$ & $\begin{array}{c}53 / 35.5444 .23 / 440 \\
320 / 225 / 15\end{array}$ \\
\hline
\end{tabular}

Table 3. Dip direction and Dip angle of acute bisector angle (stress orientation) for the conjugate joints in the NE and SW limbs in the study area

\begin{tabular}{|c|c|c|c|c|c|c|c|c|c|}
\hline \multirow{2}{*}{$\begin{array}{c}\text { acute } \\
\text { bisector } \\
\text { angle }\end{array}$} & \multirow[t]{2}{*}{$\begin{array}{l}\text { Acute } \\
\text { angle }\end{array}$} & \multicolumn{2}{|c|}{$\begin{array}{l}\text { single measurement of } \\
\text { conjugate joint }\end{array}$} & \multirow{2}{*}{$\begin{array}{l}\text { No. of } \\
\text { stations } \\
\text { in The } \\
\text { SW } \\
\text { limb }\end{array}$} & \multirow{2}{*}{$\begin{array}{c}\text { acute } \\
\text { bisector } \\
\text { angle }\end{array}$} & \multirow[t]{2}{*}{$\begin{array}{l}\text { Acute } \\
\text { angle }\end{array}$} & \multicolumn{2}{|c|}{$\begin{array}{l}\text { single measurement of } \\
\text { conjugate joint }\end{array}$} & \multirow{2}{*}{$\begin{array}{l}\text { No. of } \\
\text { stations } \\
\text { in the } \\
\text { NE } \\
\text { limb }\end{array}$} \\
\hline & & $\begin{array}{l}\text { Classific } \\
\text { ation }\end{array}$ & dip dir/dip & & & & $\begin{array}{l}\text { Classific } \\
\text { ation }\end{array}$ & dip dir/dip & \\
\hline $233.6 / 79.8$ & 42.4 & $\begin{array}{l}\text { okl }>c \\
\text { okl }>c\end{array}$ & $\begin{array}{l}302 / 79 \\
165 / 82\end{array}$ & 12 & $320.8 / 87.5$ & 64.9 & $\begin{array}{l}\mathrm{hko}_{4} \\
\mathrm{hho}_{3}\end{array}$ & $\begin{array}{c}198.28 / 87.14 \\
263.2 / 83.6\end{array}$ & 5 \\
\hline $226 / 84.4$ & 21.9 & $\begin{array}{l}\text { hol>c } \\
\text { hol>c }\end{array}$ & $\begin{array}{l}305 / 85 \\
147 / 84 \\
\end{array}$ & 13 & $226.2 / 77.2$ & 18.6 & $\begin{array}{l}\mathrm{okl}>\mathrm{c} \\
\mathrm{okl}>\mathrm{c}\end{array}$ & $\begin{array}{l}325.75 / 76.87 \\
126.75 / 77.83\end{array}$ & 18 \\
\hline $218.8 / 77.1$ & 54 & $\begin{array}{l}\mathrm{hko}_{1} \\
\mathrm{hko}_{2}\end{array}$ & $\begin{array}{l}101 / 76 \\
336 / 81\end{array}$ & 17 & $244.9 / 84.7$ & 54.5 & $\begin{array}{l}\mathrm{hko}_{2} \\
\mathrm{hko}_{1}\end{array}$ & $\begin{array}{c}307.5 / 85.16 \\
182.2 / 85.4 \\
\end{array}$ & 22 \\
\hline $124 / 84.8$ & 31.9 & $\begin{array}{l}\mathrm{hko}_{3} \\
\mathrm{hko}_{4}\end{array}$ & $\begin{array}{l}050 / 85 \\
018 / 85\end{array}$ & 23 & $215.6 / 84.1$ & 41.9 & $\begin{array}{l}\text { hko2 } \\
\text { hko1 }\end{array}$ & $\begin{array}{c}285 / 83.14 \\
146 / 86\end{array}$ & 28 \\
\hline $308.5 / 81$ & 38.6 & $\begin{array}{l}\mathrm{hko}_{4} \\
\mathrm{hko}_{3}\end{array}$ & $\begin{array}{l}010 / 82.2 \\
238 / 81.4\end{array}$ & 30 & $227.8 / 80.7$ & 38.6 & $\begin{array}{l}\mathrm{okl}>\mathrm{c} \\
\mathrm{okl}>\mathrm{c}\end{array}$ & $\begin{array}{c}337.31 / \\
81.25 \\
118.28 / \\
81.28 \\
\end{array}$ & 35 \\
\hline & & & & & $223.8 / 76.7$ & 24.5 & $\begin{array}{l}\mathrm{okl}>\mathrm{c} \\
\mathrm{okl}>\mathrm{c}\end{array}$ & $\begin{array}{c}326.37 / \\
77.12 \\
121.2 / 76.8\end{array}$ & 44 \\
\hline
\end{tabular}


Fig 1. The geographical location map of the study area

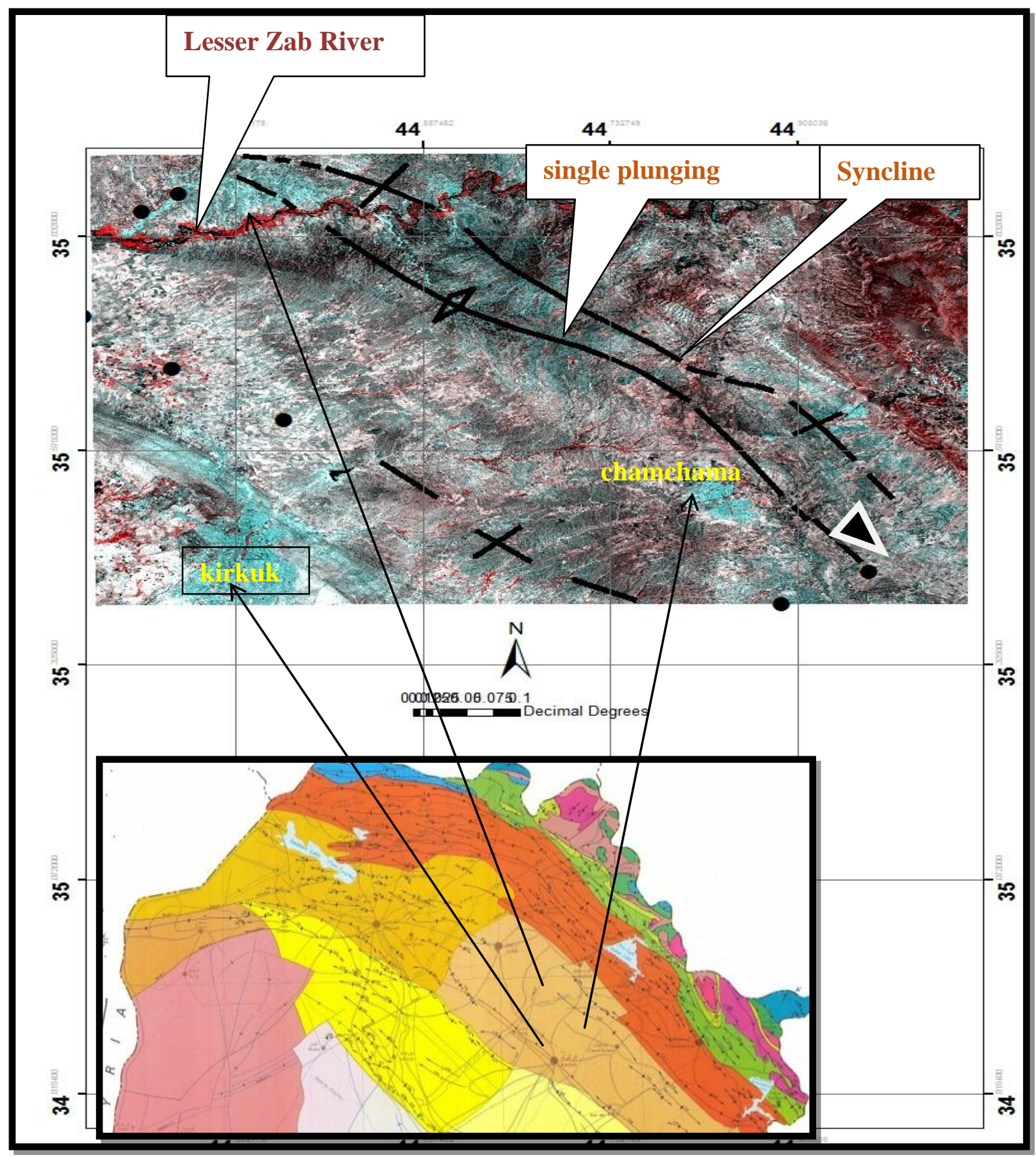


Fig 2. Tectonic map of the study area from (Al-Kadhimi, et al.,1996)

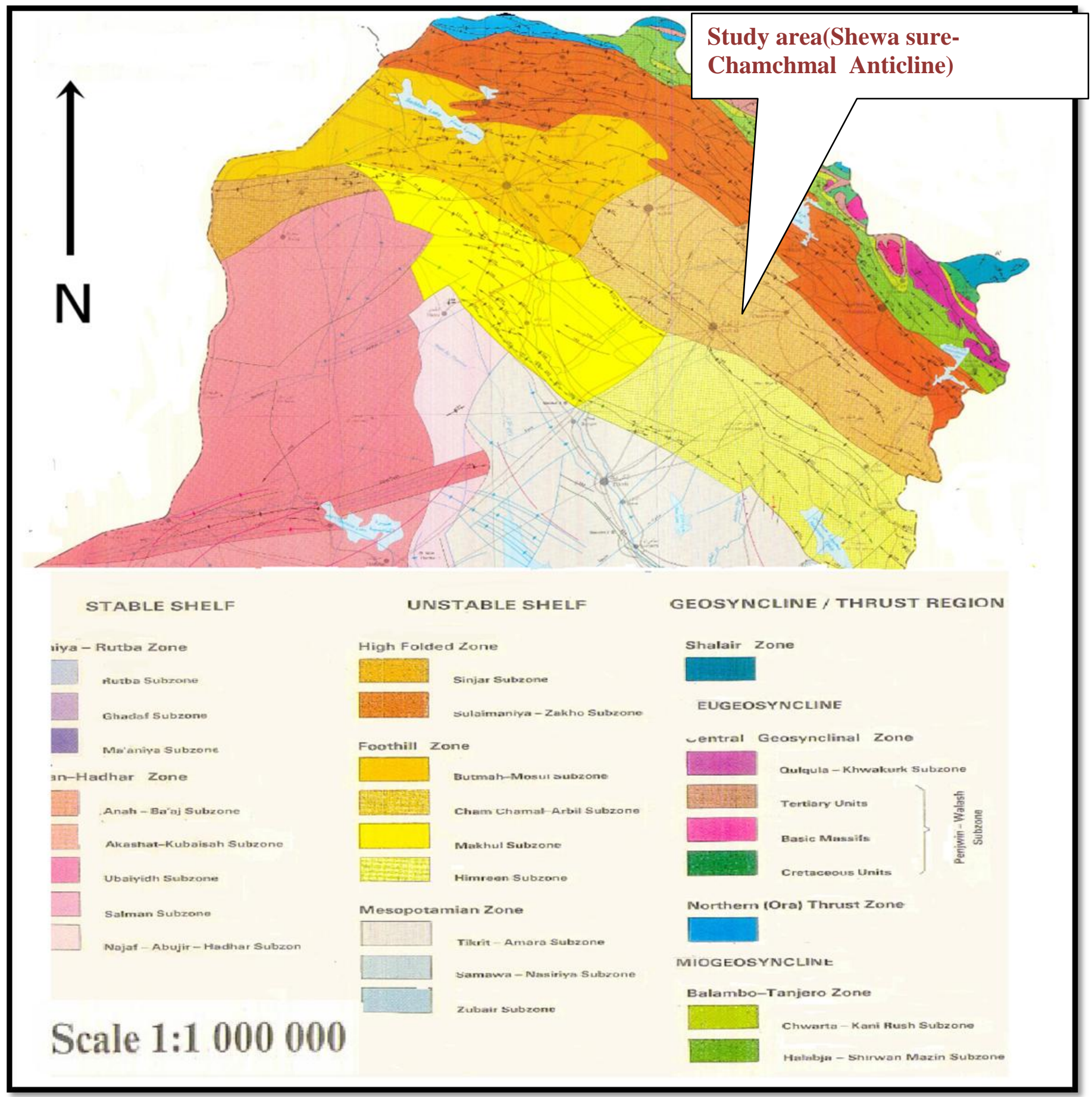


Fig 3. Geologic map of the study area from (Sissakian, 1993) showing the joints stations

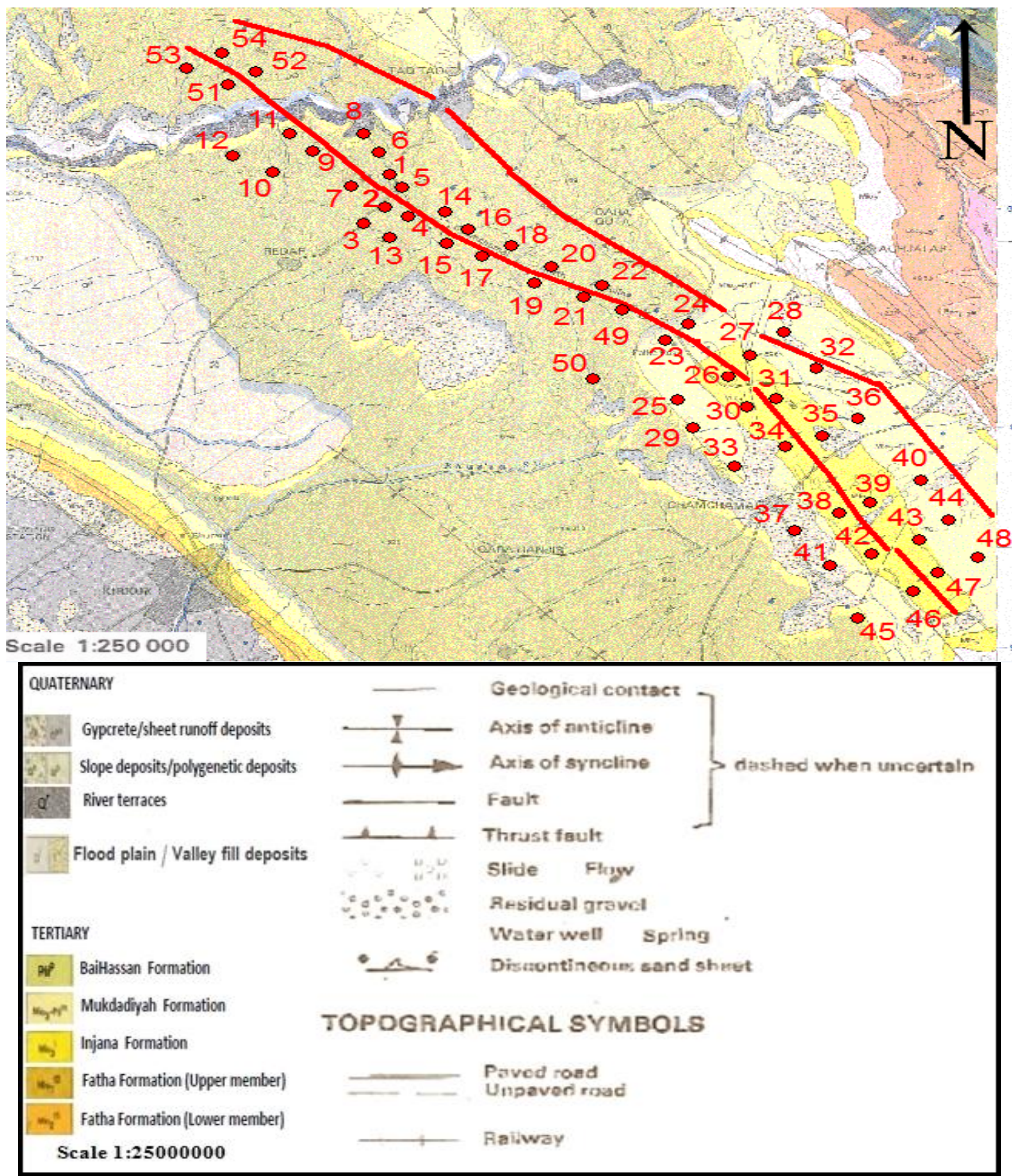


Fig 4. Stereographic projection (lower hemisphere of the measured joints,numbers represent numbers of the station

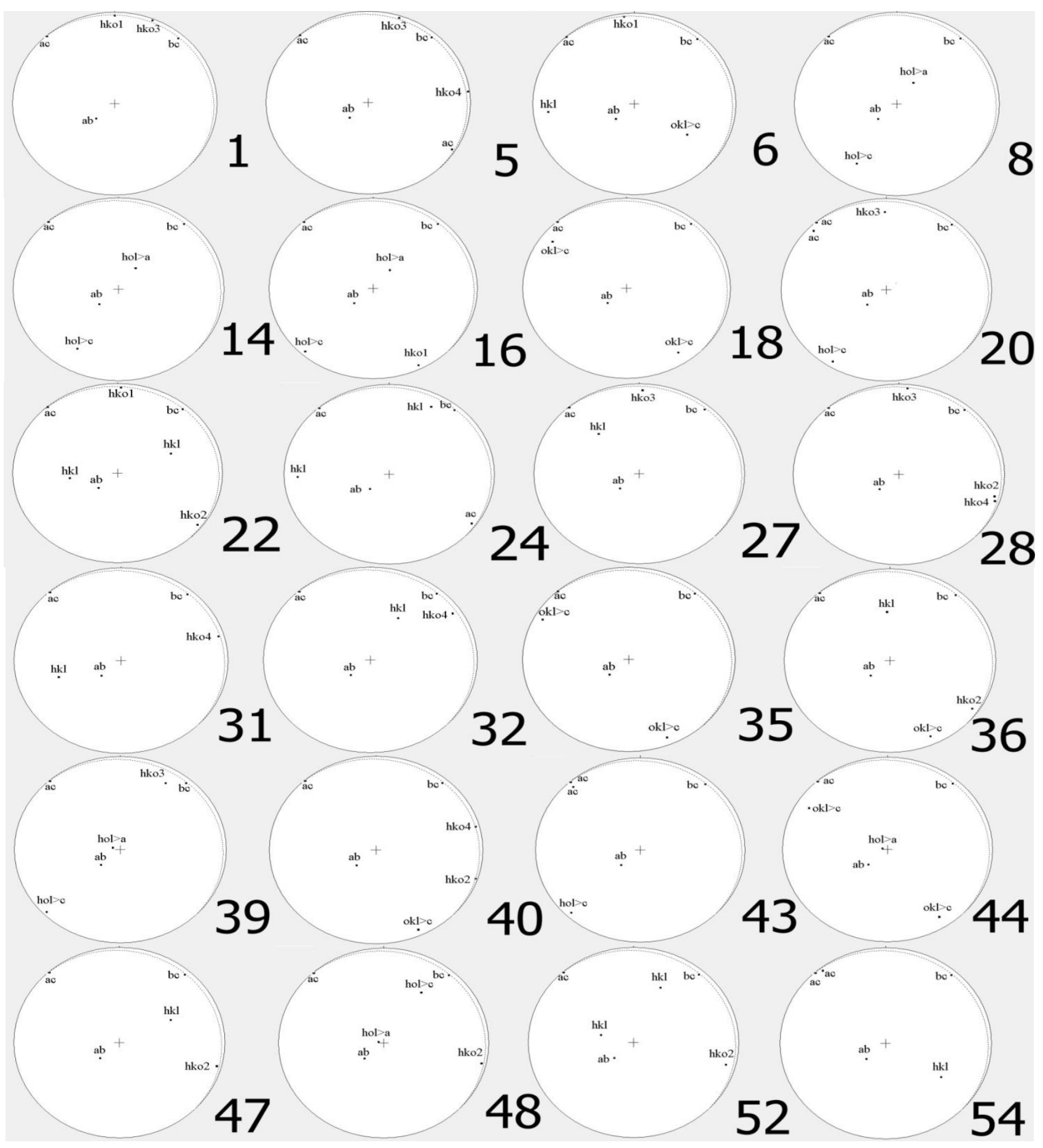


Fig 5. Stereographic projection (lower hemisphere of the measured joints,numbers represent numbers of the station in the SW limb in the study area

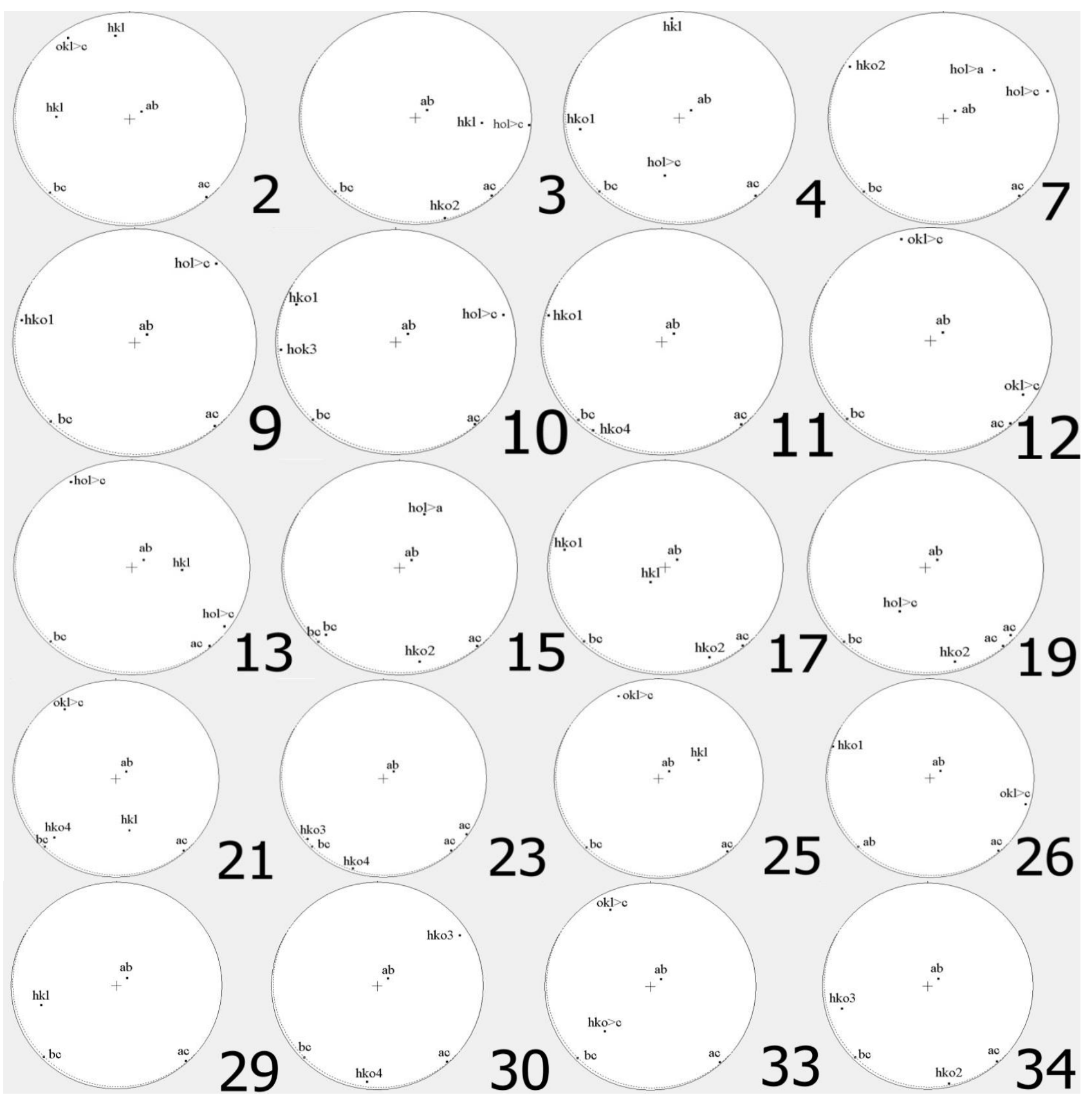




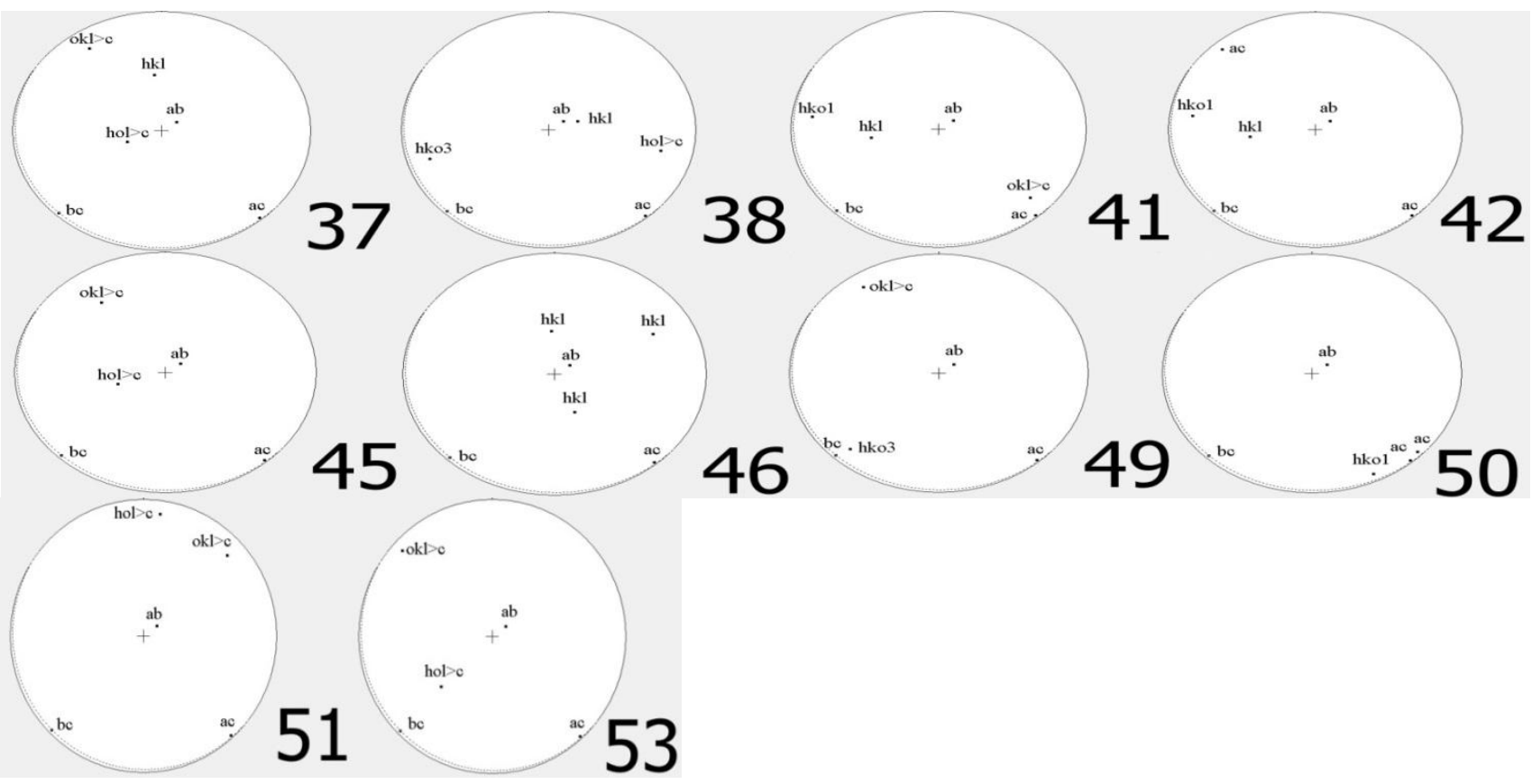

\section{Conjugate joints and stress}

The conjugate joints exist in the study area in station $(5,18,22,28,35,44)$ in the $\mathrm{NE}$ limb and in station $(12,13,17,23,30)$ in the SW.

The conjugate joint measurement provided to stereonet software and it is possible to determine the orientation and dip plunge of the acute bisector which represents the orientation of stress Table (3)

\section{Results and Discussion}

The authors classified the measured joints into tension joints and shear joints.

The shear joint in the study area are in the form of hko, hol and okl while tension joints are in the form of a ax and bc joints.

From tables (3) one can find that the acute bisected orientation (stress orientation) in the NE limb oscillate from 215 to 320 and 124 to 308 in the SW limb. Two measurement in the same mentioned limb in station 5 and 28 are nearly run with fold axis orientation see table (3). The stress orientation is nearly parallel to fold axis. In the SW limb a single measurement of conjugate joint run nearly with fold axis.

From above the main stress orientation is NE-SW which coincides with Zagros stress trend.
The mentioned orientation is responsible for fold development in north and NE Iraq as the previous works clarify. Thus the studied fold is formed as the folds formed in NE Iraq.

\section{Conclusion and recommendation}

1-The calculated stress orientation is run comfortable with Zagros stress and the adjacent calculated stress.

2-The present study illustrates that other orientation of stress runs with fold axis. This could be of tension along type.

3-The existence of high percent of hkl joints could be due to gypsum and anhydride beds within Fatha Formation.

4- The fold takes the present shape during Pliocene or may be post Pliocene. This is guided by the presence Pliocene sediments exposures of Mukdadiya and BiaHassan Formations.

It is worth to mentioned that more structural studies are needed to support the present work especially if we know that the study area is virgins from structural studies.

\section{References}

Al-Abdulla, N.M.A.O., 2009. Comparative structural study of the brittle failure structures between the upper Cretaceous and Tertiary rocks in SpiRais and 
Shaikhan Anticline, $\mathrm{N}$ Iraq, unpublished. MSc. Thesis, univ. of Mosul, Mosul, Iraq.

Al-Assawi, A.G.N. 2010. The structural and Tectonic analysis of Bashiqa and Al-Fadhlya anticline-North Iraq, unpublished. Thesis, univ. of Mosul, Mosul Iraq.

Al-Brifkani, M.J.N. 2008. Structural and Tectonic Analysis of the northern Turst Zone (East Khabour River) in Iraq, unpub, ph.D. Thesis, University of Mosul, Mosul, 174p ( In Arabic) .

Al-Kadhimi, A.M., Sissakian, V.K., Fattah, A.S., and Diekran, D.B. 1996. Tectonic Map of Iraq, Printed and Published by GEOSERV, Iraq, 42p.

Al-Khatony, S.E.M. 2009. The structural analysis and tectonic interpretation of Shaikhan Anticline Northern Iraq, unpub. M.Sc. Thesis, University of Mosul, Mosul-Iraq, (In Arabic).

Alleghanian Orogeny. 2007. J. Structural Geol., 29: $774 \mathrm{e} 786$.

Al-Sumaidaie, M.AH.K. 2010. Structural and Tectonic study of Brifka anticline northern Iraq, unpublished thesis, univ. of Mosul, Mosul, Iraq.

Barno, J.M.G. 2001. Structural analysis of Kosrat anticline and its tectonic implication, NE Iraq, unpublished, phD. thesis, univ. of Baghdad, Baghdad, Iraq.

Buday, T. and Jassim, S.Z. 1980. The regional geology of Iraq, Vol.1, Stratigraphy and Paleogeography, Dar Al-Kutib Pub. House, Mosul, Iraq, 445 p.

Buday, T., Jassim, S.Z., Kassab, I., M. and Abbas, M.J. 1987. The Regional Geology of Iraq Vol.2, Tectonism, Magmatism \& Metamorphism, state Establishment of geological Survey and Mineral investigation, Baghdad, Iraq, $352 \mathrm{p}$.

Dyer, R. 1988. Using joint interactions to estimate paleostress ratios, J. Structural Geol., Vol. 10, No. 7, pp. 685 to 699 .
Engelder, T. 1982. Reply to a comment by A.E. Scheidegger on "Is there a genetic relationship between selected regional joints and contemporary stress within the lithospher of North America?" Engelder. Tectonic, 1, pp465-470.

Engelder, T. and Geiser, P. 1980. On the Use of Regional Joints as Trajectories of Paleostress Fields During the Development of the Appalachian Plateau-New York, J. Geophysical.

Hancock, P.L. 1985. Brittle microtectonics: principles and practice. J. Structural Geol., Vol.7, No.3, pp.437-457, Vol.85, No.B11, pp.6319-6341.

Hancock, P.L. 1991. Determining contemporary stress direction from neotectonic joint system, Philos. Trans. R. Sic. London, 337, pp29-40

Lash, G.G. \& Engelder, T. 2007. Jointing within the outer arc of a forebulge at the onset.

McConaughy, D.T. \& Terry Engelder, T. 2000. Joint initiation in bedded clastic rocks, J. Structural Geol., 23: 203-221.

Pollard, D.D., and Aydin, A. 1988. Progress in understanding jointing over the past Century, Geol. Soc. Am. Bull., 100, pp. 1181-1204.

Shihab, A.T. 1999. Structural Analysis using remote sensing and aGis Techniques in the high folded Zone between Harrison and Bradost Anticline, NE Iraq, unpublished. Thesis, univ. of Baghdad, Baghdad, Iraq.

Sissakian, V.K. 2000. Geological map of Iraq. Sheets No.1, Scale1:1000000, 3rd edit. Geosurv, Baghdad, Iraq.

Zhao, M., Jacobi, R.D. 1996. Formation of regional cross-fold joints in the northern Appalachian Plateau, Joint J. Structural Geol., Vol. 19, No. 6, PP. 817 to 834.

\section{How to cite this article:}

Duraid B. Deikran and Aryan S. Baban. 2017. Geometrical Classification and Estimation of Stresses of the Joints in the Shewa Sur-Anticline, NE Iraq. Int.J.Curr.Res.Aca.Rev. 5(1), 121-133.

doi: http://dx.doi.org/10.20546/ijcrar.2017.501.015 